\title{
Melatonin attenuates hLRRK2-induced sleep disturbances and synaptic dysfunction in a Drosophila model of Parkinson's disease
}

\author{
XICUI SUN ${ }^{1 *}$, DONGZHI RAN ${ }^{2 *}$, XIAOFENG ZHAO ${ }^{3}$, YI HUANG $^{1}$, SIMEI LONG $^{1}$, \\ FENGYIN LIANG ${ }^{1}$, WENYUAN GUO ${ }^{1}$, FREDERICK C. NUCIFORA Jr. ${ }^{4}$, HUAIYU GU ${ }^{2}$, XILIN LU $^{1}$, \\ LING CHEN $^{1}$, JINSHENG ZENG ${ }^{1}$, CHRISTOPHER A. ROSS ${ }^{4,5}$ and ZHONG PEI ${ }^{1}$
}

\begin{abstract}
${ }^{1}$ Department of Neurology, National Key Clinical Department and Key Discipline of Neurology, The First Affiliated Hospital; ${ }^{2}$ Department of Anatomy and Neurobiology, Zhongshan School of Medicine, Sun Yat-Sen University, Guangzhou, Guangdong 510080; ${ }^{3}$ Key Laboratory, Liaocheng Hospital, Liaocheng, Shandong 252000;

${ }^{4}$ Division of Neurobiology, Department of Psychiatry; ${ }^{5}$ Division of Neurobiology, Department of Psychiatry, Departments of Neurology, Neuroscience and Pharmacology and Program in Cellular and Molecular Medicine, Johns Hopkins University School of Medicine, Baltimore, MD 21287, USA
\end{abstract}

Received March 19, 2015; Accepted January 21, 2016

DOI: $10.3892 / \mathrm{mmr} .2016 .4991$

\begin{abstract}
Sleep problems are the most common non-motor symptoms in Parkinson's disease (PD), and are more difficult to treat than the motor symptoms. In the current study, the role of human leucine-rich repeat kinase 2 (hLRRK2), the most common genetic cause of PD, was investigated with regards to sleep problems, and the therapeutic potential of melatonin in hLRRK2-associated sleep problems was explored in Drosophila. hLRRK2 was selectively expressed in the mushroom bodies (MBs) in Drosophila and sleep patterns were measured using the Drosophila Activity Monitoring System. MB expression of hLRRK2 resulted in sleep problems, presynaptic dysfunction as evidenced by reduced miniature excitatory postsynaptic current (mEPSC) and excitatory postsynaptic potential (EPSP) frequency, and excessive synaptic plasticity such as increased axon bouton density. Treatment with melatonin at $4 \mathrm{mM}$ significantly attenuated the sleep problems and rescued the reduction in $\mathrm{EEPSC}$ and EPSP frequency in the hLRRK2 transgenic flies. The present study demonstrates that MB expression of hLRRK2 in flies recapitulates the clinical features of the sleep disturbances in PD, and that melatonin attenuates hLRRK2-induced sleep disorders and synaptic
\end{abstract}

Correspondence to: Professor Zhong Pei, Department of Neurology, National Key Clinical Department and Key Discipline of Neurology, The First Affiliated Hospital, Sun Yat-Sen University, 58 Zhongshan Road II, Guangzhou, Guangdong 510080, P.R. China E-mail: peizhong@mail.sysu.edu.cn

${ }^{*}$ Contributed equally

Key words: LRRK2, Drosophila, mushroom body, sleep pattern, electrophysiology properties, melatonin dysfunction, suggesting the therapeutic potential of melatonin in PD patients carrying LRRK2 mutations.

\section{Introduction}

Parkinson's disease is the second most common neurodegenerative disease and affects nearly $1 \%$ of the worldwide population over $65(1,2)$. Traditionally, PD is regarded as the most common movement disorder due to the fact that the majority of patients with PD present with predominantly motor symptoms including tremor, rigidity, slow movements and gait problems (3). However, non-motor symptoms are also very common in PD. For example, sleep problems, the most frequent non-motor symptoms, affect $65-95 \%$ of patients (4-7). Furthermore, the non-motor symptoms may equally or more adversely affect the quality life of patients with PD.

Although the etiology of PD is not clear, it is generally believed that both genetic susceptibility and environmental factors contribute to the pathogenesis of PD. Mutations in leucine-rich repeat kinase 2 (LRRK2) gene is the most common genetic cause of familial and sporadic PD (8-11). Similar to sporadic late-onset PD, sleep problems are the major complaints of patients with PD who have LRRK2 mutations. It has been reported that sleep disruptions are present in 60-98\% of patients with LRRK2-associated PD (12). Thus, studying the role of LRRK2 in sleep disturbances may generate new insights into the pathophysiology of PD, providing therapeutic options for the management of sleep disorders in LRRK2-associated PD.

The fruit fly, Drosophila melanogaster, has been increasingly used to model neurologic diseases due to the similarity in the nervous system between fruit flies and humans (13-17). Several LRRK2 transgenic flies have been created to study the role of LRRK2 mutations $(18,19)$. These LRRK2 transgenic flies recapitulate several key features of human PD including locomotor dysfunction and selective loss of dopaminergic neurons (20). However, little is known about sleep disorders 
in these LRRK2 transgenic flies. Sleep in Drosophila is regulated by the MBs. MB output regulates the sleep through different downstream neurons, while ablation of MB output leads to sleep disturbances $(21,22)$.

Clinically, sleep problems are more difficult to treat than motor symptoms in PD due to the multifactorial causes of sleep disturbances. Furthermore, current treatments for PD sleep disorders are often associated with undesirable adverse effects. Melatonin is an endogenous regulator of the sleep/wake cycle. Melatonin secretion patterns change in PD patients, and treatment with melatonin replenishes the inadequate melatonin levels, relieving the sleep symptoms (23). In addition to its sleep-promoting effects, melatonin is an effective antioxidant. The neuroprotective activity of melatonin has been well documented in a range of models of PD $(24,25)$. However, these beneficial effects of melatonin have not been explored in LRRK2-associated PD. In the present study, transgenic flies were generated to express human (h)LRRK2 in the MBs. Transgenic flies expressing hLRRK2 were observed to exhibit sleep problems such as sleep fragmentation during the night. Additionally, hLRRK2 was observed to disrupt the presynaptic function in the Kenyon cells (KCs) in the MB and increase bouton density. Furthermore, administration of melatonin significantly attenuated the sleep problems and rescues the presynaptic dysfunction in the hLRRK2 transgenic flies.

\section{Materials and methods}

Generation of human LRRK2 transgenic flies. The transgenic flies were constructed to bear the different hLRRK 2 constructs [wild type (WT) and G2019S] under the control of a yeast upstream activating sequence (UAS). A green fluorescent protein (GFP) XbaI-EcoRI fragment was first ligated into the pUAST vector to generate a UAS-GFP construct. Flag tagged hLRRK 2 was then inserted between the KpnI and BglII sites of the UAS-GFP vector. The constructs were injected into $\mathrm{w}^{1118}$ embryos (Bloomington Drosophila Stock Center, Bloomington, IN, USA; $n=6$ in each group) to obtain transformant lines. Two transgenic lines each were generated for UAS-GFP-hLRRK2 and UAS-GFP-hLRRK2-G2019S. The hLRRK2 expression levels were examined by western blot analysis using anti-Flag staining. OK107-GAL4 was used to selectively express UAS-GFP-hLRRK2 and UAS-GFP-hLRRK2-G2019S in the MBs. The phenotypic characterization was conducted on hLRRK2-WT and hLRRK2-G2019S lines. $\mathrm{w}^{1118}$ served as a negative control. Drosophila were grown on standard cornmeal medium at $25^{\circ} \mathrm{C}$ under $12 / 12 \mathrm{~h}$ light/dark (L/D) conditions. For melatonin treatment, flies were transferred to regular fly food containing $4 \mathrm{mM}$ melatonin.

Western blotting. The heads of adult flies (3-7 days post eclosion) were collected and homogenized in radioimmunoprecipitation assay lysis buffer (EMD Millipore, Billerica, MA, USA) containing a protease inhibitor cocktail (Roche Diagnostics $\mathrm{GmbH}$, Mannheim, Germany). Following centrifugation at $8,000 \mathrm{x}$ g for $15 \mathrm{~min}$ at $4^{\circ} \mathrm{C}$, the supernatants were subjected to Bradford protein assays (Beyotime Institute of Biotechnology, Haimen, China) to ensure equal protein loading $(50 \mu \mathrm{g})$ and resolved on $8 \%$ sodium dodecyl sulfate-polyacrylamide gel electrophoresis gel, and then transferred onto polyvinylidene difluoride membranes (0.45 mm; EMD Millipore). The membranes were blocked in Tris-buffered saline- $0.1 \%$ Tween 20 containing 5\% nonfat milk for $1 \mathrm{~h}$ at room temperature and then probed with primary antibodies overnight at $4^{\circ} \mathrm{C}$ and secondary antibodies for $1 \mathrm{~h}$ at room temperature. The primary antibodies used were as follows: a mouse monoclonal anti-Flag-tag antibody (1:2,000; Sigma-Aldrich, St. Louis, MO, USA; cat. no. F3165) and mouse monoclonal anti- $\beta$-actin (1:3,000; ProteinTech Group, Inc., Chicago, IL, USA; cat. no. 60008-01). The secondary antibody was horseradish peroxidase-conjugated goat anti-mouse antibody (1:6,000; EarthOx Life Sciences, Millbrae, CA, USA; cat. no. E030110-01). Protein was detected using chemiluminescence reagents (Beyotime Institute of Biotechnology).

Sleep assays. Individual female virgin flies (3-7 days post eclosion; $\mathrm{n}=32$ in each group) were transferred into monitor tubes $(5 \times 65 \mathrm{~mm})$ containing 5\% sucrose and 2\% agar media at one end, enabling the continuous recording of behavior over a number of days. Locomotor activity was recorded by the Drosophila Activity Monitoring System (DAMS; TriKinetics, Inc., Waltham, MA, USA). Sleep was defined as periods of 5 min without recorded activity. Data were collected for 3 days. Experiments were performed in an incubator at a temperature of $25 \pm 1^{\circ} \mathrm{C}$ and a relative humidity of $60 \pm 5 \%$. Lights were turned on at zeitgeber time (ZT)0 (circadian time 06:00) and off at ZT12 (circadian time 18:00).

Electrophysiological recordings from $\mathrm{KCs}$ in isolated whole brains. All brains were obtained from flies 1-3 days in age. The entire brain including the optic lobes was removed from the head. The dissected brains were then mounted in an RC-26 perfusion chamber (Warner Instruments, LLC, Hamden, CT, USA) containing the recording solution bubbled with $95 \%$ $\mathrm{O}_{2}$ and $5 \% \mathrm{CO}_{2}(2 \mathrm{ml} / \mathrm{min})$ throughout the experiments with the ventral surface of the brain facing up (26). The standard external solution contained (in $\mathrm{mM}$ ): $101 \mathrm{NaCl}, 1 \mathrm{CaCl}_{2}$, $4 \mathrm{MgCl}_{2}, 3 \mathrm{KCl}, 5$ glucose, $1.25 \mathrm{NaH}_{2} \mathrm{PO}$, and $20.7 \mathrm{NaHCO}_{3}$, at $\mathrm{pH} 7.2$, osmolarity 250 Osm. Recording pipettes were fabricated from capillary glass using a four stage micropipette puller, and had tip resistances of 15-20 M $\Omega$, when filled with the intracellular solution of the following composition (in $\mathrm{mM})$ : $102 \mathrm{~K}$-gluconate, $17 \mathrm{KCl}, 0.94$ ethylene glycol tetraacetic acid, 8.5 4-(2-hydroxyethyl)-1-piperazineethanesulfonic acid, $1.7 \mathrm{MgCl}_{2}, 17 \mathrm{NaCl}$, at $\mathrm{pH} \mathrm{7.2,} \mathrm{osmolarity} 235 \mathrm{Osm}$. Pipettes were targeted to GFP-labeled KCs in the MBs. Current-clamp ( $n=6$ in each group) and voltage-clamp ( $n=6$ in each group) recordings were performed using patch-clamp electrodes. Giga-ohm seals were achieved prior to recording in an on-cell configuration, followed by whole-cell configuration while in voltage-clamp mode. Recordings were made at room temperature, and only a single $\mathrm{KC}$ neuron was examined in each brain. Excitatory postsynaptic potential (EPSP) frequency and amplitude were recorded using whole-cell current-clamp. For measurements of cholinergic miniature excitatory postsynaptic current (mEPSC), neurons were held at $-75 \mathrm{mV}$ followed by whole-cell configuration in voltage clamp, tetrodotoxin $(1 \mu \mathrm{M})$ was added to the external solution to block voltage-gated sodium currents and $\gamma$-aminobutyric acid-ergic synaptic currents were blocked by picrotoxin 
Table I. Sleep behavior of flies expressing hLRRK2-WT and hLRRK2-G2019S (with and without melatonin treatment), driven by the OK107-GAL4-mediated expression of upstream activating sequence transgenes and $\mathrm{w}^{1118}$.

\begin{tabular}{lccccccc}
\hline & \multicolumn{3}{c}{ Light phase } & & & Dark phase & \\
\cline { 2 - 3 } Group & $\begin{array}{c}\text { Sleep bout } \\
\text { (number) }\end{array}$ & $\begin{array}{c}\text { Mean bout } \\
\text { length (min) }\end{array}$ & Sleep (min) & $\begin{array}{c}\text { Sleep bout } \\
\text { (number) }\end{array}$ & $\begin{array}{c}\text { Mean bout } \\
\text { length (min) }\end{array}$ & $\begin{array}{c}\text { Sleep } \\
(\mathrm{min})\end{array}$ \\
\hline Control & $8.281 \pm 0.743$ & $141.997 \pm 19.541^{\mathrm{b}}$ & $579.484 \pm 9.063^{\mathrm{b}}$ & $11.500 \pm 1.067^{\mathrm{b}}$ & & $41.449 \pm 1.170^{\mathrm{c}}$ & $470.687 \pm 11.698^{\mathrm{b}}$ \\
WT & $9.938 \pm 0.853^{\mathrm{d}}$ & $85.648 \pm 9.493^{\mathrm{e}}$ & $542.078 \pm 9.282^{\mathrm{d}}$ & $13.859 \pm 1.778$ & & $39.627 \pm 4.256$ & $387.625 \pm 12.625$ \\
G2019S & $14.875 \pm 1.162^{\mathrm{f}}$ & $74.109 \pm 10.287^{\mathrm{f}}$ & $523.797 \pm 11.293^{\mathrm{e}}$ & $17.906 \pm 0.867^{\mathrm{f}}$ & $22.975 \pm 1.463^{\mathrm{f}}$ & $348.125 \pm 10.718^{\mathrm{e}}$ \\
G2019S (M) & $9.094 \pm 0.714^{\mathrm{c}}$ & $121.475 \pm 16.085^{\mathrm{b}}$ & $552.313 \pm 9.083^{\mathrm{a}}$ & $15.359 \pm 0.892^{\mathrm{a}}$ & $26.100 \pm 2.219$ & $310.016 \pm 13.212$ \\
\hline
\end{tabular}

Data are presented as the mean \pm standard error of the mean $(n=32,3$ independent experiments performed). Differences between means were determined by one-way analysis of variance followed by the Newman-Keuls multiple comparison post hoc test. ${ }^{\mathrm{a}} \mathrm{P}<0.05$, ${ }^{\mathrm{b}} \mathrm{P}<0.01$, ${ }^{\mathrm{c}} \mathrm{P}<0.001$ vs. the hLRRK2- G2019S mutant without melatonin and ${ }^{\mathrm{d}} \mathrm{P}<0.05,{ }^{\mathrm{e}} \mathrm{P}<0.01,{ }^{\mathrm{f}} \mathrm{P}<0.001$ vs. the control ( ${ }^{1118}$ ) group. hLRRK2, human leucine-rich repeat kinase 2; WT, wild type; G2019S, glycine 2019 serine.

$(10 \mu \mathrm{M})$. mEPSC amplitudes $<20 \mathrm{pA}$ were detected. All electrophysiological recordings were conducted using a BX51WI upright microscope (Olympus Corporation, Tokyo, Japan). Data were acquired by MultiClamp 700B amplifier and an Axon Digidata1440A (Molecular Devices, LLC, Sunnyvale, CA, USA), and were filtered at $5 \mathrm{kHz}$ using a built-in filter and digitized at $5 \mathrm{kHz}$. Data were analyzed offline using Clampfit 10.2 (Molecular Devices, LLC).

Biocytin staining and two-photon laser scanning fluorescence microscopy. To examine the morphology of the recorded single neurons, post hoc staining with biocytin was utilized. In these cells ( $\mathrm{n}=7$ in each group), $0.4 \%$ biocytin was added to the internal pipette solution. Following electrophysiological recordings, the brain was fixed in phosphate buffered $4 \%$ formaldehyde for $3 \mathrm{~h}$ on ice. The brains were then washed 5 times with phosphate-buffered saline-Tween 20 on the orbital shaker for $10 \mathrm{~min}$ at room temperature and then blocked in blocking buffer (0.1 M Tris-HCl, $0.1 \%$ Triton X-100, 10\% goat serum) for $3 \mathrm{~h}$ on ice. Brains were incubated in $\mathrm{Cy} 3$ (1:100 dilution) for $24 \mathrm{~h}$ at $4^{\circ} \mathrm{C}$.

A Leica TCS SP5 microscope (Leica Microsystems $\mathrm{GmbH}$, Wetzlar, Germany) with a 40x objective was used to acquire optical slices through the MBs. The position of the soma was determined by both the position of electrode tip and the intense biocytin staining. An argon laser provided the excitation line at $546 \mathrm{~nm}$ with the gate for the multiplier opened between 500 and $600 \mathrm{~nm}$. Slices of the brain ( 120) were generated at a thickness of $1 \mu \mathrm{m}$ per step (from the top of the soma to bottom of the dendrite in the z-axis through the tissue), at a $1024 \times 2048 \mathrm{~Hz}$ scanning speed. Two photon images were saved as lif, the preferred file format for image processing using Imarisx64 software, version 7.2.1 (Bitplane AG, Zurich, Switzerland), which were used to create 3D optical volumes of the neuronal dendrites, from which the synaptic boutons can be detected.

Data analysis. All statistical analysis was conducted using SPSS 19.0 software (IBM SPSS, Armonk, NY, USA). The Shapiro-Wilks test was used to determine the normality of data. The normally distributed data were analyzed by 2-tailed, unpaired one-way analysis of variance followed by the Newman-Keuls multiple comparison post hoc test. Data are presented as the mean \pm standard error of the mean. $\mathrm{P}<0.05$ was considered to indicate a statistically significant difference.

\section{Results}

$M B$ expression of hLRRK2 did not result in gross morphological alterations. hLRRK2 was selectively expressed in the MBs using the OK107 promoter to target GAL4 expression in all lobes of the MBs. In these transgenic flies, hLRRK2 was expressed in all MB lobes as indicated by the GFP fluorescence (Fig. 1A). Structurally, MB lobes remained intact in flies expressing either hLRRK2-WT or hLRRK2-G2019S. The size and thickness of the MB lobes were similar between control and hLRRK2 flies (Fig. 1A). Confocal microscopy did not reveal any cell loss and axonal degeneration of KCs in all the examined LRRK2 files compared with the control. Western blotting demonstrated that the protein expression was stable and at similar levels between the hLRRK2-WT and hLRRK2-G2019S flies (Fig. 1B). Taken together, these results indicate that expression of hLRRK2 in the MBs does not result in any significant morphological alterations.

$M B$ expression of $h L R R K 2$ disrupted normal sleep patterns. To explore whether MB expression of hLRRK2 results in sleep problems, sleep patterns were investigated in hLRRK2 flies. Compared with the control flies, the bout number of hLRRK2-WT flies were increased ( $\mathrm{P}=0.035$; Fig. 2A) and the mean length of each sleep episode in the light phase were significantly reduced (Fig. 2B, P=0.002), however, not in the dark phase (Fig. 2D and E). By contrast, the expression of hLRRK2-G2019S markedly increased bout number (counts; Fig. 2A, P<0.001; Fig. 2D, P<0.001) and reduced bout length (min) in both the light and dark phases (Fig. 2B, $\mathrm{P}<0.001$; Fig. 2E, $\mathrm{P}<0.001)$. Furthermore, the total sleep (min) of the hLRRK2-G2019S mutant flies was significantly reduced (Fig. 2C, P=0.007; Fig. 2F, $\mathrm{P}=0.004$ ), however, not in 
A

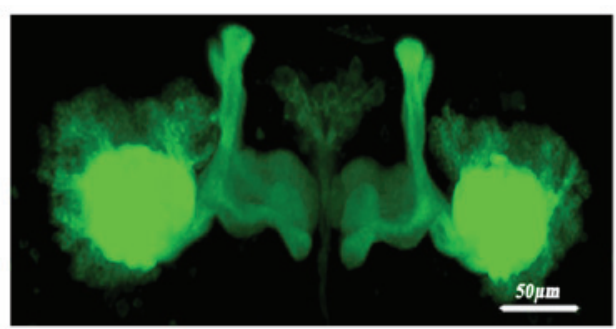

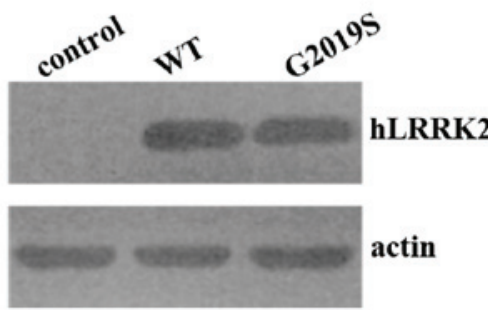

Figure 1. hLRRK2 was expressed in the MBs in flies. (A) Image showing MB expression of hLRRK2 and green fluorescent protein driven by GAL4-OK107 (Scale bar, $50 \mu \mathrm{m}$ ). (B) Image of western blot showing the expression of hLRRK2 in the MBs. hLRRK2, human leucine-rich repeat kinase 2; MBs; mushroom bodies; WT, wild type; G2019S, glycine 2019 serine.
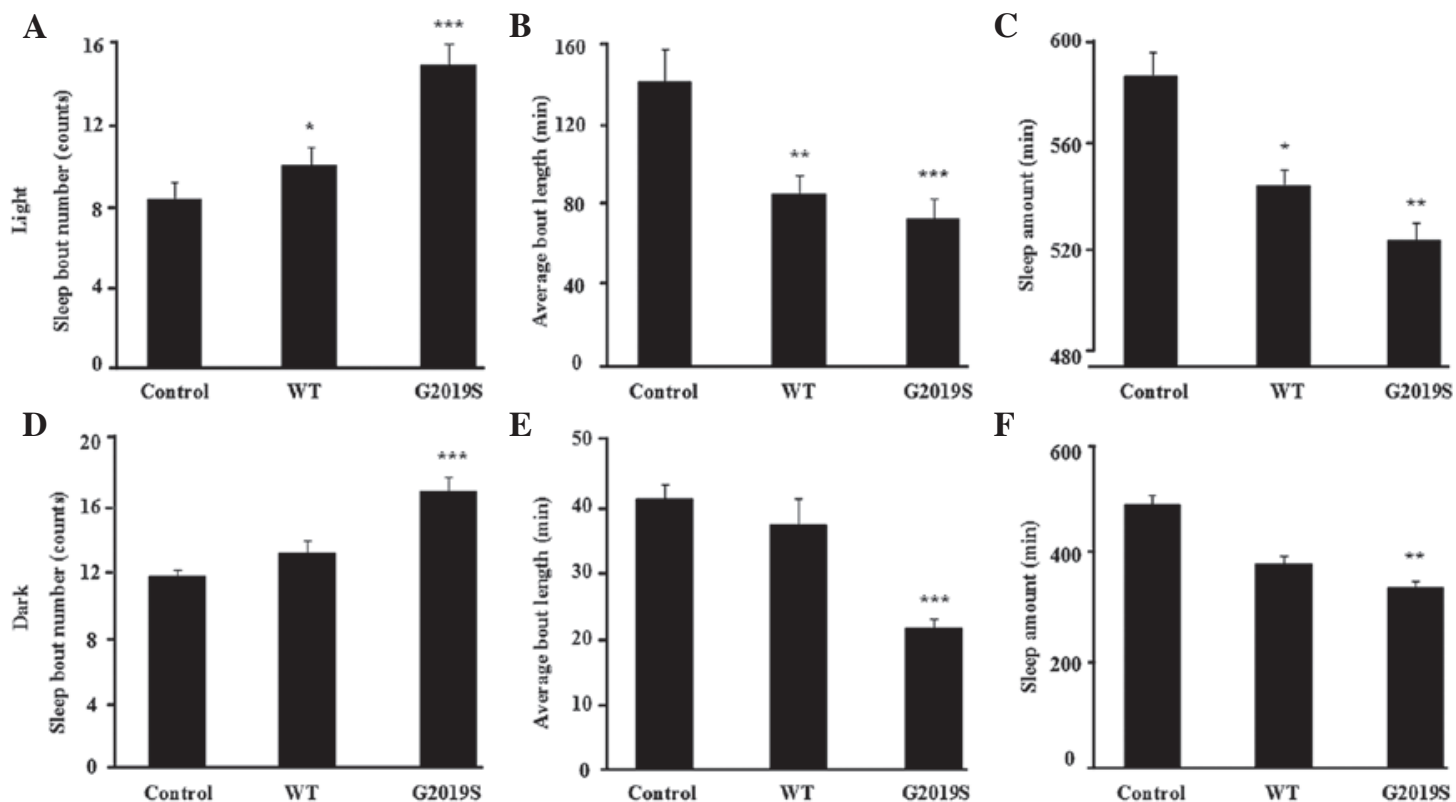

F

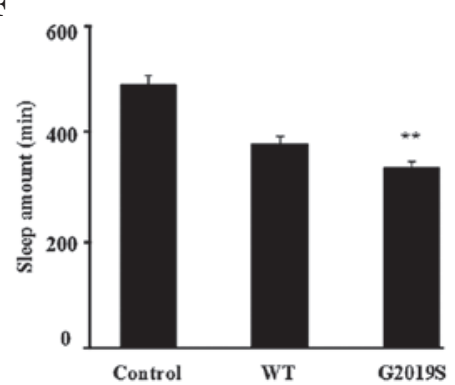

Figure 2. Sleep behavior of flies expressing hLRRK2-WT and hLRRK2-G2019S, driven by the OK107-GAL4-mediated expression of UAS transgenes and $\mathrm{w}^{1118}$. Expression of the hLRRK2-G2019S mutation in young flies (3 days after eclosion) affected (A and D) the average number of sleep episodes (sleep bout number), (B and E) the average length of sleep episodes (bout length) and (C and F) the sleep amount (total sleep) to a greater extent than $\mathrm{w}^{1118}$ and hLRRK2-WT expression during the (A-C) light and (D-F) dark phase. Data are presented as the mean \pm standard error of the mean from 32 animals that were individually recorded in each experiment, from a minimum of 3 independent experiments. Differences between means were determined by one-way analysis of variance followed by the Newman-Keuls multiple comparison post hoc test. ${ }^{*} \mathrm{P}<0.05,{ }^{* *} \mathrm{P}<0.01,{ }^{* * *} \mathrm{P}<0.001$ vs. the control $\left(\mathrm{w}^{1118}\right)$ group. hLRRK2, human leucine-rich repeat kinase 2; WT, wild type; G2019S, glycine 2019 serine.

the hLRRK2-WT flies compared with the control group (Fig. 2C and F). The sleep behavior data is presented in Table I. Collectively, the data suggest that MB expression of hLRRK2 disrupted normal sleep patterns in flies, predominantly by increasing arousal during sleep and reducing total sleep time.

$M B$ expression of hLRRK2 resulted in presynaptic dysfunction. The synaptic functions of KCs, such as membrane excitability, are thought to be critical for sleep regulation. To investigate whether hLRRK2 modulates the synaptic function of $\mathrm{KCs}$, whole-cell current-clamp recording (Fig. 3A) and voltage-clamp recordings in $\mathrm{MB} \mathrm{KCs}$ was performed (Fig. 3D). During the recordings, the frequency and amplitude of the EPSP and mEPSC were detected, with the amplitude and frequency of the mEPSC indicating the postsynaptic function and presynaptic function, respectively. When the holding potential was $-75 \mathrm{mV}$ in voltage-clamp mode, and the mean resting potential was $-60 \pm 1.25 \mathrm{mV}$ in current-clamp mode, none of the examined KCs exhibited spontaneous firing activities, however, some exhibited subthreshold spontaneous activity in normal control flies (Fig. 3A and D). The EPSP amplitude in WT $(1.624 \pm 0.1759 \mathrm{mV} ; \mathrm{P}=0.572)$ and $\mathrm{G} 2019 \mathrm{~S}$ $(0.954 \pm 0.169 \mathrm{mV}, \mathrm{P}=0.994)$ were not significantly different from the control flies $(1.091 \pm 0.219 \mathrm{mV}$; Fig. 3B). By contrast, the EPSP frequency in WT $(12.300 \pm 1.174 \mathrm{~Hz}, \mathrm{P}=0.008)$ and G2019S $(6.850 \pm 1.053 \mathrm{~Hz}, \mathrm{P}=0.004)$ were significantly lower compared with the control flies $(25.525 \pm 3.957 \mathrm{~Hz}$; Fig. 3C). Similarly, the mEPSCs frequency of WT $(0.388 \pm 0.0557 \mathrm{~Hz}$; $\mathrm{P}<0.05)$ and G2019S $(0.3101 \pm 0.462 \mathrm{~Hz}$; $<<0.01$; Fig. $3 \mathrm{~F})$ were significantly lower than the control $\mathrm{w}^{1118}(0.778 \pm 0.078$; Fig. 3F), however, the mEPSCs amplitude of WT and G2019S indicated no significant difference compared with the control $\mathrm{w}^{1118}$ (Fig. 3E). However, the amplitude but not the frequency of either mEPSCs or EPSPs were similar between the hLRRK2 flies and control flies (Fig. 3B and E), indicating that postsynaptic but not presynaptic function remained intact in hLRRK2 flies. Taken together, these results indicate that hLRRK2 
A

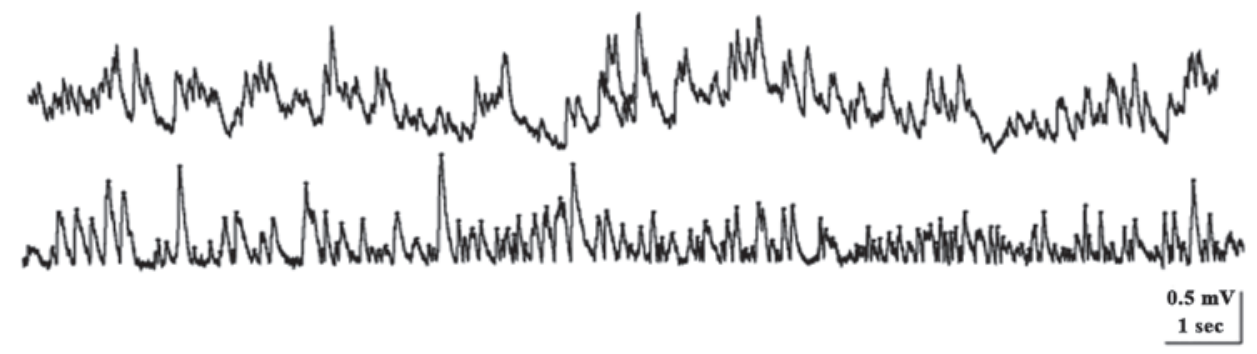

B

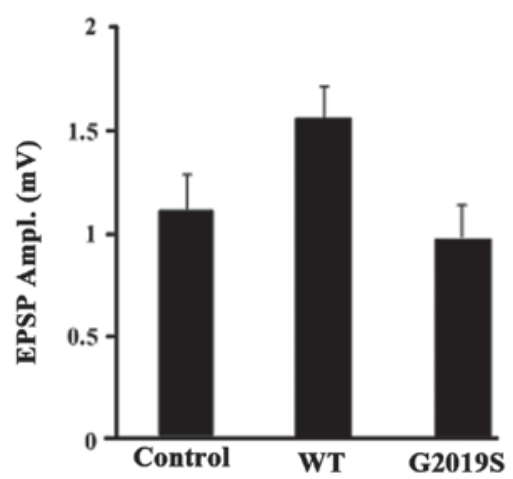

C

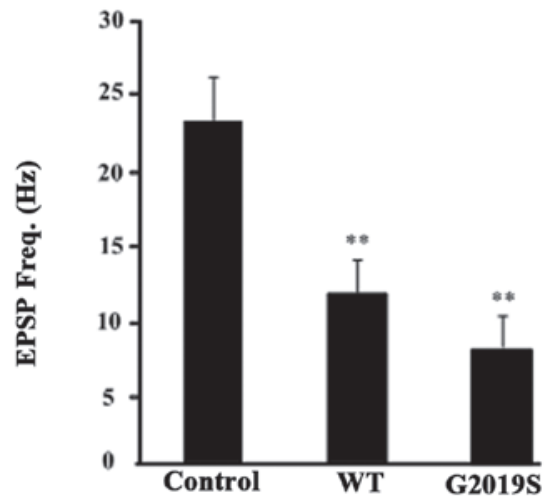

D

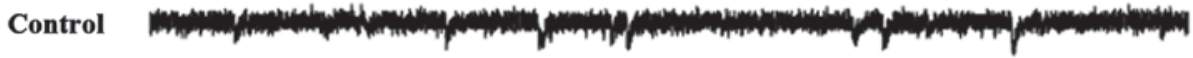

WT

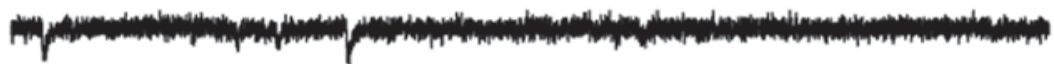

G2019S

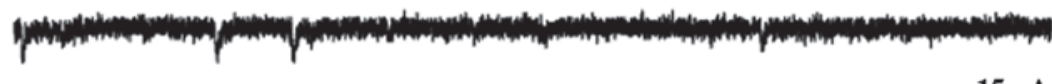

$200 \mathrm{~ms}$

E

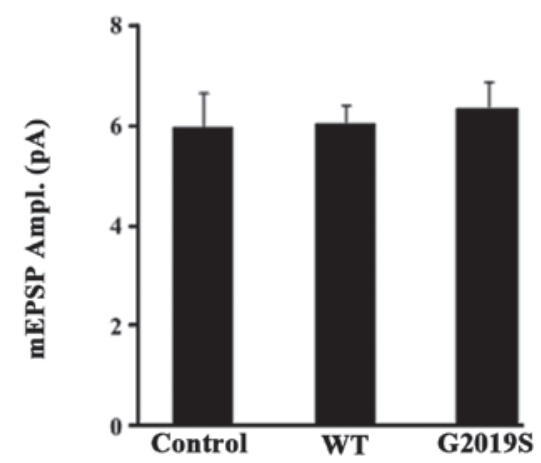

F

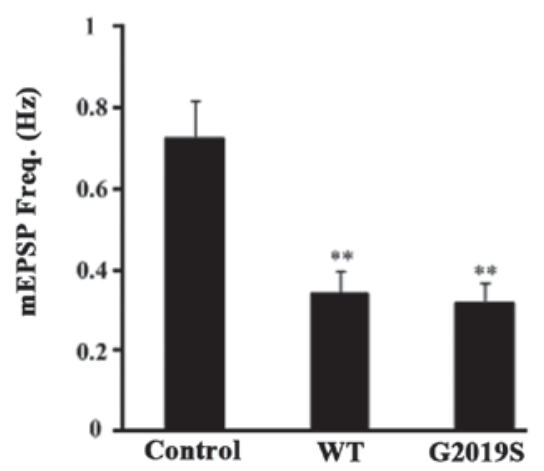

Figure 3. mEPSC and EPSP are affected in hLRRK2 transgenic flies. (A) Representative waves of the EPSP. (B) The amplitude of the EPSP was not affected in hLRRK2 transgenic flies. (C) The frequency of the EPSP was reduced in hLRRK2 transgenic flies. (D) Representative waves of the mEPSC. (E) The amplitude of the mEPSC was not affected in hLRRK2 transgenic flies. $(\mathrm{F}) \mathrm{mEPSC}$ frequency was reduced in hLRRK2 transgenic flies. ${ }^{* *} \mathrm{P}<0.01 \mathrm{vs}$. the control $\left(\mathrm{w}^{1118}\right.$ ) group. Data are presented as the mean \pm standard error of the mean, $n=6$. mEPSC, miniature excitatory postsynaptic current; EPSP, excitatory postsynaptic potential; hLRRK2, human leucine-rich repeat kinase 2; WT, wild type; G2019S, glycine 2019 serine.

expression results in presynaptic dysfunction, as evidenced by the reduction in the cellular excitability of KCs.

$M B$ expression of hLRRK2-G2019S increases the density of synaptic boutons. To investigate whether hLRRK2-G2019S affects synaptic plasticity, the synaptic bouton density (synaptic terminals) was investigated. Biocytin staining was used to label the synaptic boutons of the KCs in the MBs (Fig. 4Aa) and Fig. 4Ab presents a single $\mathrm{KC}$ neuron. To identify the density of neurons accurately and efficiency, an imaging method of the KCs was tested, based on two-photon laser scanning fluorescence microscopy, which enabled the visualization of the density of KCs synaptic boutons. The density of boutons was significantly higher in hLRRK2-G2019S mutant flies $(0.2010 \pm 0.0238 \mu \mathrm{m} ; \mathrm{P}=0.025)$ while the density of boutons remained unaltered in hLRRK2-WT flies $(0.1145 \pm 0.0465 \mu \mathrm{m} ; \mathrm{P}=0.89)$ compared with the $\mathrm{w}^{1118}$ control flies $(0.1246 \pm 0.0145 \mu \mathrm{m}$; Fig. 4B). Thus, these results demon- 
A
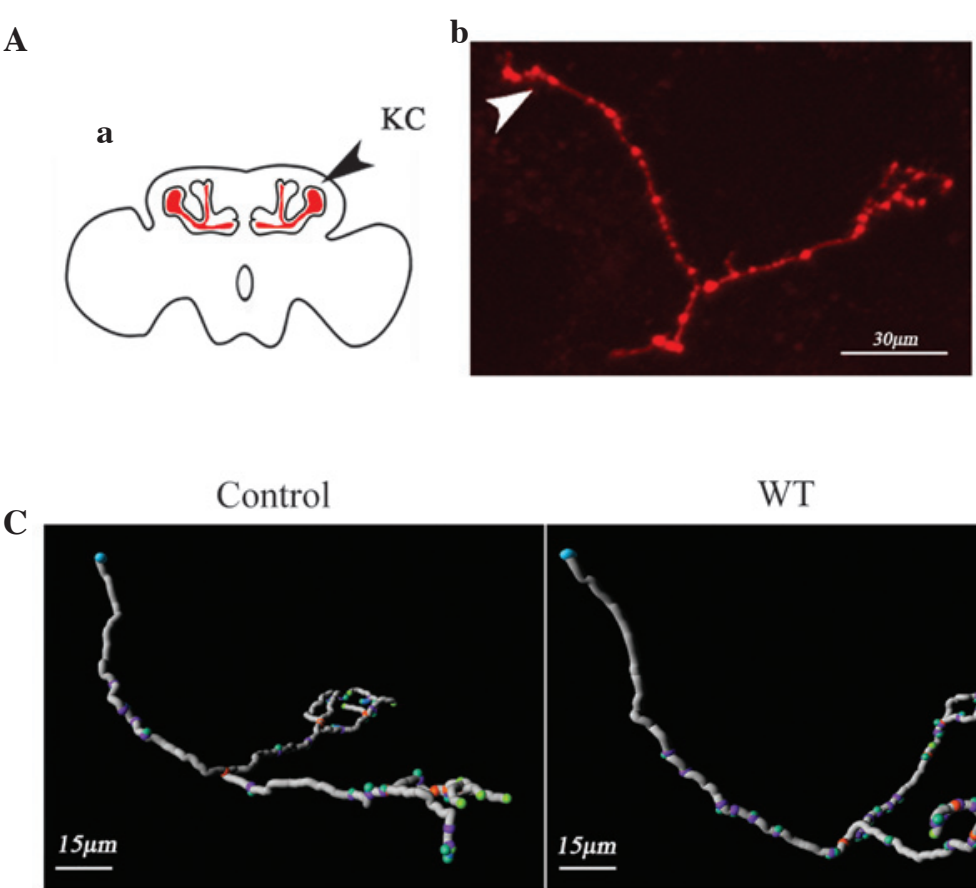

Control

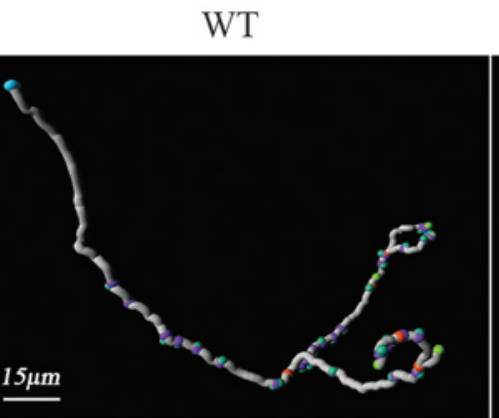

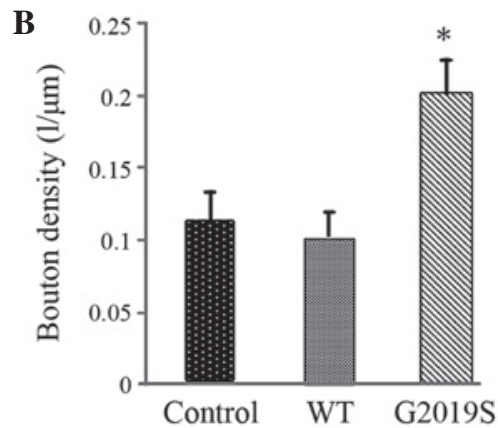

Figure 4. Axon bouton density is altered in hLRRK2-G2019S mutant flies. (A) Representative images of KC axons. (a) Dissected Drosophila whole brain with the mushroom bodies indicated in red. (b) A single KC indicated with biocytin. (B) The density of the axon boutons was increased in hLRRK2-G2019S mutant flies. (C) Representative images of the 3D reconstruction of the axonal boutons. Scale bar, $15 \mu \mathrm{m},{ }^{*} \mathrm{P}<0.05$ vs. the control w, $\mathrm{w}^{1118}$ group, $\mathrm{n}=7$. Data are presented as the mean \pm standard error of the mean. hLRRK2-G2019S, human leucine-rich repeat kinase 2-glycine 2019 serine; KC, Kenyon cell; WT, wild type.
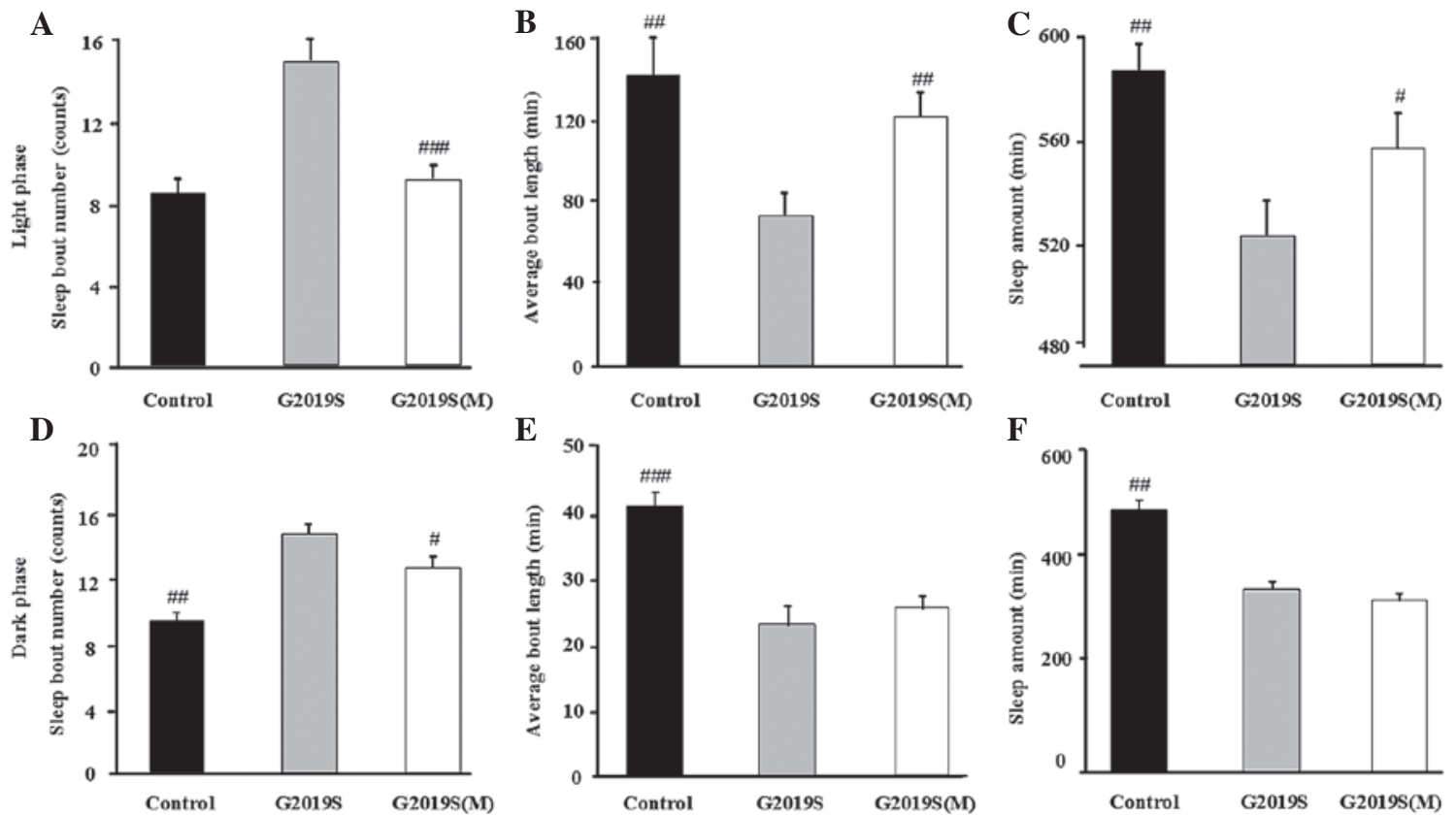

Figure 5. Melatonin attenuates sleep disorders in hLRRK2-G2019S mutant flies. (A) Melatonin reduced the number of light phase of sleep in hLRRK2-G2019S mutant flies. (B) Melatonin increased the light phase length of bouts of sleep in hLRRK2-G2019S mutant flies. (C) Melatonin increased the total amount of light phase sleep in hLRRK2-G2019S mutant flies. (D) Melatonin reduced the number of dark phase bouts of sleep in hLRRK2-G2019S mutant flies. (E) Melatonin did not affect the length of dark phase bouts of sleep in hLRRK2-G2019S mutant flies. (F) Melatonin did not affect the dark phase sleep amount in hLRRK2-G2019S mutant. Data are presented as the mean \pm standard error of the mean $(\mathrm{n}=32)$. ${ }^{\# P}<0.05,{ }^{\# \#} \mathrm{P}<0.01,{ }^{\# \# \#} \mathrm{P}<0.001$ vs. the hLRRK2-G2019S mutant without melatonin treatment. hLRRK2-G2019S, human leucine-rich repeat kinase 2-glycine 2019 serine; M, melatonin.

strate that hLRRK2-G2019S induces excessive synaptic plasticity (Fig. 4C).

Melatonin attenuates sleep disturbances and rescues presynaptic dysfunction in hLRRK2 flies. Whether mela- tonin attenuates hLRRK2-associated sleep problems and the relationship between sleep disorder and synaptic dysfunction was investigated. It was observed that melatonin significantly attenuated the hLRRK2-induced increase in light and dark phase sleep bouts (Fig. 5A and D), and melatonin attenuated the 

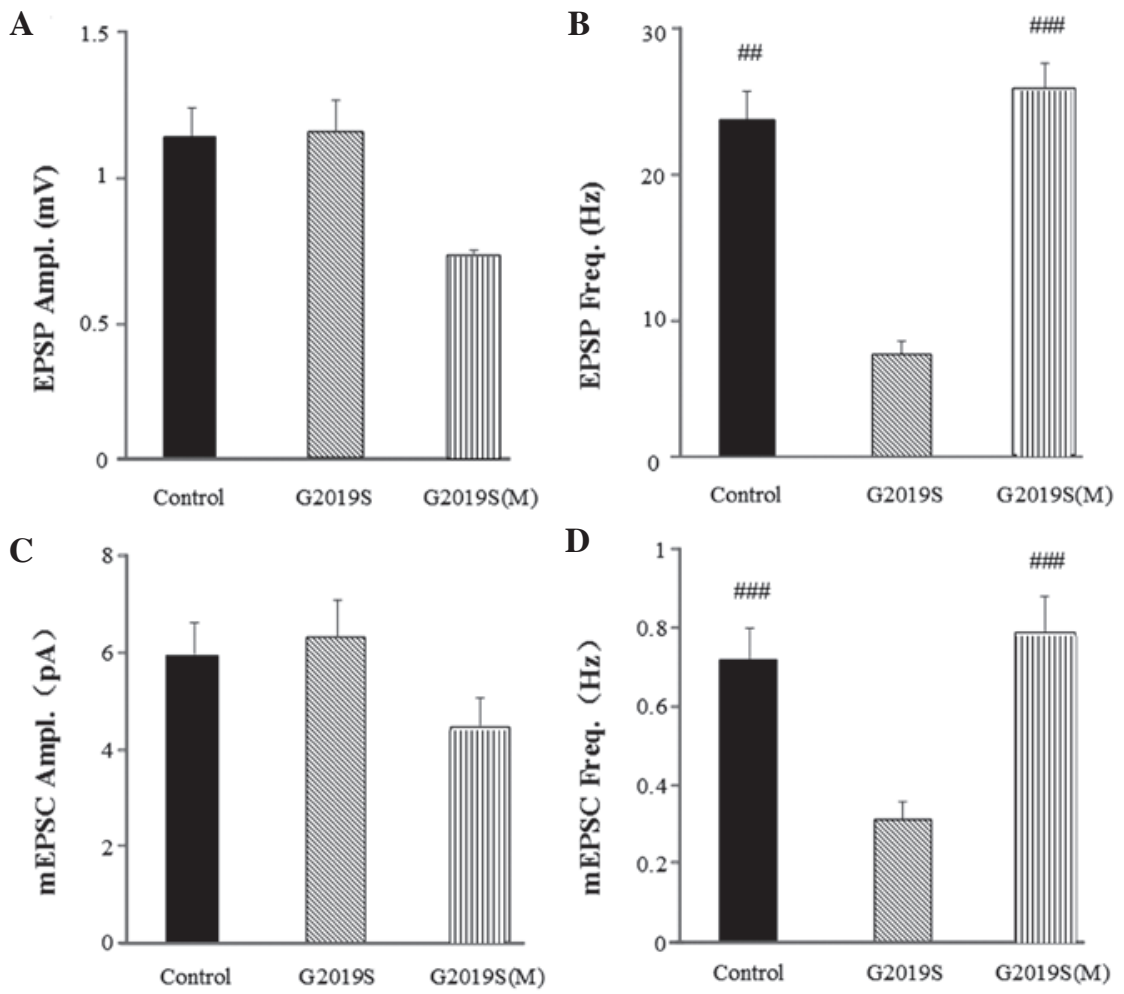

Figure 6. Melatonin increases mEPSC and EPSP frequency in hLRRK2-G2019S mutant flies. (A) Melatonin did not affect EPSP amplitude. (B) Melatonin increased EPSP frequency. (C) Melatonin did not affect mEPSC amplitude. (D) Melatonin increased mEPSC frequency. Data are presented as the mean \pm standard error of the mean $(\mathrm{n}=6) .{ }^{\#} \mathrm{P}<0.05 ;{ }^{\# \#} \mathrm{P}<0.01,{ }^{\# \# \#} \mathrm{P}<0.001$ vs. the hLRRK2-G2019S mutant without melatonin treatment. mEPSC, miniature excitatory postsynaptic current; EPSP, excitatory postsynaptic potential; hLRRK2-G2019S, human leucine-rich repeat kinase 2-glycine 2019 serine; M, melatonin.

hLRRK2-induced reduction in the mean bout length and the amount of sleep in the light phase compared with the G2019S flies without melatonin (Fig. 5B and C). However, the mean bout length and the amount of sleep in the light phase were not altered (Fig. 5E and F). All sleep behavior data is presented in Table I. Furthermore, the EPSP and mEPSC frequencies in the hLRRK2 flies were restored to the levels of the control group following melatonin treatment compared with the flies without melatonin treatment (Fig. 6). The EPSP frequency of G2019S flies treated with melatonin was restored to $26.022 \pm 6.534 \mathrm{~Hz}$ $(\mathrm{P}<0.001$; Fig. 6B), which was significantly different from the EPSP frequency of the G2019S flies without melatonin $(6.850 \pm 1.953 \mathrm{~Hz})$. The mEPSC frequency in the G2019S flies treated with melatonin was restored to $0.785 \pm 0.104 \mathrm{~Hz}$ $(\mathrm{P}<0.001$; Fig. 6D), which was significantly different from the mEPSC frequency of the G2019S flies without melatonin $(0.310 \pm 0.046 \mathrm{~Hz})$. Amplitude differences in mEPSCs suggest changes to postsynaptic function and frequency differences suggest changes to presynaptic function. The results from the present study indicated neither the EPSP (Fig. 6A) or mEPSC (Fig. 6C) amplitudes were altered in the hLRRK2 flies, with or without melatonin treatment. However, the frequency of EPSP (Fig. 6B) and mEPSC (Fig. 6D was altered with melatonin, thus, suggesting that melatonin improves sleep problems and presynaptic dysfunction in hLRRK2 flies.

\section{Discussion}

Mutations in LRRK2 are the most common genetic cause for familial and sporadic PD (27). Thus, studying the role of hLRRK2 in PD is important to further understand the pathophysiology of PD. The present study reports that the expression of hLRRK2 in the MBs induced sleep disorders in flies. hLRRK2-induced sleep problems were associated with reduced frequency of EPSPs and increased synaptic bouton density, indicating the important role of hLRRK2-induced synaptic dysfunction in sleep disorders. Furthermore, melatonin significantly attenuated hLRRK2-induced sleep problems and rescued hLRRK2-induced synaptic dysfunction, suggesting a potential clinical application of melatonin in patients with PD carrying LRRK2 mutations. Sleep problems are a major contributor to impairment in PD, with $80-90 \%$ of patients with PD experiencing disturbances of sleep patterns $(28,29)$. Although the manifestations of sleep problems vary, sleep fragmentation is one of the most common sleep complaints of patients with PD (30-32). Consistently, MB expression of hLRRK2 in flies resulted in sleep fragmentation as indicated by the increase in arousal during sleep. Thus, the transgenic flies expressing hLRRK2 in the MBs are able to recapitulate the sleep disturbances observed in clinical PD. The underlying causes of sleep problems in PD are complex. Previous studies have demonstrated that a large proportion of sleep problems are associated with the involvement of non-dopaminergic brain regions with multiple neurotransmitter deficiencies such as cholinergic system degeneration (33-36). The MBs serve a central role in sleep regulation in flies (22). The MBs are a paired brain structure, and are composed of small neurons known as KCs. The synaptic functions of the KCs are critical for sleep regulation (21). In the present study, expression of hLRRK2 
in the MBs did not result in any gross morphological damage however, reduced cholinergic synaptic mEPSC and EPSP frequency was observed, suggesting a negative modulation of hLRRK2 on presynaptic properties. Notably, in addition to sleep problems, the synaptic bouton density was increased in hLRRK2-G2019S flies. Synaptic boutons are the presynaptic terminals that contain neurotransmitters stored in synaptic vesicles. The increase in the number of synaptic boutons means greater levels of neurotransmitters present, which in turn increases the probability of the neurotransmitter release. Thus, the increase in synaptic bouton density seems contradictory to the negative modulation of hLRRK2 on synapses. This may be explained by the inhibitory effect of hLRRK2 on synaptic efficacy. Synaptic efficacy is the capacity of a presynaptic input to influence postsynaptic output. Previously, hLRRK2 has been reported to disrupt synaptic vesicle trafficking and distribution within the bouton (37). By doing so, hLRRK2 may prevent the release of neurotransmitters from the vesicles in the synaptic boutons and reduce synaptic efficacy. Furthermore, the increase in the number of synaptic boutons may lead to synaptic stress due to the increase in synaptic boutons occupying greater space and demanding increased cellular supply to synapses (38). As a result, low synaptic efficacy and synaptic stress may further disrupt synaptic homeostasis, leading to an exaggeration of hLRRK2-induced sleep problems.

The primary function of sleep is to restore brain energy metabolism, with sleep problems increasing energy consumption and exaggerating the metabolic disturbances in PD. In this regard, treatment of sleep disturbances with appropriate drugs may help to not only to solve sleep problems, but also to prevent the progression of PD. In the present study, melatonin significantly attenuated hLRRK2-induced sleep problems and rescued the reduced cholinergic synaptic mEPSC and EPSP frequencies, however, had no effect on the increase in synaptic bouton density. These results suggest that the beneficial effects of melatonin may be associated with the promotion of synaptic transmission. The direct action of melatonin on synaptic transmission remains inconclusive due to inconsistent results from different studies (39). Alternatively, the promotion of synaptic transmission may be explained by the antioxidant role of melatonin. Reactive oxygen species (ROS) have been reported to be involved in vesicular neurotransmitter release (40). At presynaptic sites, certain essential proteins responsible for the exocytosis of neurotransmitters such as synaptosomal-associated protein, $25 \mathrm{kDa}$ are vulnerable to ROS attack due to their chemical structures (41). The oxidation of these key proteins impairs the neurotransmitter release machinery. Indeed, ROS scavengers have been reported to prevent ROS attack and enhance synaptic transmission (41). Thus, melatonin may reduce ROS and attenuate hLRRK2-induced synaptic dysfunction. As a result, melatonin may break the cycle involving sleep disturbances and metabolic stress, and prevent the progression of LRRK2-associated PD. However, the precise mechanisms underlying the beneficial effects of melatonin require further study. Nevertheless, the present study highlights the potential of melatonin as a candidate neuroprotective agent with sleep-promoting properties in future clinical trials for patients with PD carrying hLRRK2 mutations.

\section{Acknowledgements}

The current study was supported by grants from the National Natural Science Foundation of China (grant no. 81371255), the Doctoral Program of Higher Education of China (grant no. 20110171110058) and the Guangdong Province Science and Technology Department Project (grant nos. 2011B050400031 and 2012B031800107).

\section{References}

1. Braak H, Del Tredici K, Rüb U, de Vos RA, Jansen Steur EN and Braak E: Staging of brain pathology related to sporadic Parkinson's disease. Neurobiol Aging 24: 197-211, 2003.

2. Dauer W and Przedborski S: Parkinson's disease: Mechanisms and models. Neuron 39: 889-909, 2003.

3. Parkinson J: An essay on the shaking palsy. 1817.J Neuropsychiatry Clin Neurosci 14: 223-236; discussion 222, 2002.

4. Comella CL: Sleep disorders in Parkinson's disease: An overview. Mov Disord 22 (Suppl 17): S367-S373, 2007.

5. Van Hilten JJ, Weggeman M, Van der Velde EA, Kerkhof GA, Van Dijk JG and Roos RA: Sleep, excessive daytime sleepiness and fatigue in Parkinson's disease. J Neural Transm Park Dis Dement Sect 5: 235-244, 1993.

6. Mehta SH, Morgan JC and Sethi KD: Sleep disorders associated with Parkinson's disease: Role of dopamine, epidemiology and clinical scales of assessment. CNS Spectr 13 (3 Suppl 4): 6S-11S, 2008.

7. Nausieda PA, Weiner WJ, Kaplan LR, Weber S and Klawans HL: Sleep disruption in the course of chronic levodopa therapy: An early feature of the levodopa psychosis. Clin Neuropharmacol 5: 183-194, 1982.

8. Cookson MR: The role of leucine-rich repeat kinase 2 (LRRK2) in Parkinson's disease. Nat Rev Neurosci 11: 791-797, 2010.

9. Greggio E and Cookson MR: Leucine-rich repeat kinase 2 mutations and Parkinson's disease: Three questions. ASN Neuro 1: e00002, 2009.

10. Paisán-Ru1́z C, Jain S, Evans EW, Gilks WP, Simón J, van der Brug M, López de Munain A, Aparicio S, Gil AM, Khan N, et al: Cloning of the gene containing mutations that cause PARK8-linked Parkinson's disease. Neuron 44: 595-600, 2004.

11. Zimprich A, Müller-Myhsok B, Farrer M, Leitner P, Sharma M, Hulihan M, Lockhart P, Strongosky A, Kachergus J, Calne DB, et al: The PARK8 locus in autosomal dominant parkinsonism: Confirmation of linkage and further delineation of the disease-containing interval. Am J Hum Genet 74: 11-19, 2004.

12. Berg D, Schweitzer KJ, Leitner P, Zimprich A, Lichtner P, Belcredi P, Brüssel T, Schulte C, Maass S, Nägele T, et al: Type and frequency of mutations in the LRRK2 gene in familial and sporadic Parkinson's disease*. Brain 128: 3000-3011, 2005.

13. Dawson TM, Ko HS and Dawson VL: Genetic animal models of Parkinson's disease. Neuron 66: 646-661, 2010.

14. Harbison ST and Sehgal A: Quantitative genetic analysis of sleep in Drosophila melanogaster. Genetics 178: 2341-2360, 2008.

15. Huber R, Hill SL, Holladay C, Biesiadecki M, Tononi G and Cirelli C: Sleep homeostasis in Drosophila melanogaster. Sleep 27: 628-639, 2004.

16. Koh K, Evans JM, Hendricks JC and Sehgal A: A Drosophila model for age-associated changes in sleep: Wake cycles. Proc Natl Acad Sci USA 103: 13843-13847, 2006.

17. Mackay TF and Anholt RR: Of flies and man: Drosophila as a model for human complex traits. Annu Rev Genomics Hum Genet 7: 339-367, 2006.

18. Li T, Yang D, Sushchky S, Liu Z and Smith WW: Models for LRRK2-linked parkinsonism. Parkinsons Dis 2011: 942412, 2011.

19. Liu Z, Wang X, Yu Y, Li X, Wang T, Jiang H, Ren Q, Jiao Y, Sawa A, Moran T, et al: A Drosophila model for LRRK2-linked parkinsonism. Proc Natl Acad Sci USA 105: 2693-2698, 2008.

20. Lee SB, Kim W, Lee S and Chung J: Loss of LRRK2/PARK8 induces degeneration of dopaminergic neurons in Drosophila. Biochem Biophys Res Commun 358: 534-539, 2007.

21. Joiner WJ, Crocker A, White BH and Sehgal A: Sleep in Drosophila is regulated by adult mushroom bodies. Nature 441 : 757-760, 2006 
22. Pitman JL, McGill JJ, Keegan KP and Allada R: A dynamic role for the mushroom bodies in promoting sleep in Drosophila. Nature 441: 753-756, 2006.

23. Fertl E, Auff E, Doppelbauer A and Waldhauser F: Circadian secretion pattern of melatonin in Parkinson's disease. J Neural Transm Park Dis Dement Sect 3: 41-47, 1991.

24. Olakowska E, Marcol W, Kotulska K and Lewin-Kowalik J: The role of melatonin in the neurodegenerative diseases. Bratisl Lek Listy 106: 171-174, 2005.

25. Polimeni G, Esposito E, Bevelacqua V, Guarneri C and Cuzzocrea S: Role of melatonin supplementation in neurodegenerative disorders. Front Biosci (Landmark Ed) 19: 429-446, 2014

26. Gu H and O'Dowd DK: Whole cell recordings from brain of adult Drosophila. J Vis Exp 6: 248, 2007.

27. Thaler A, Ash E, Gan-Or Z, Orr-Urtreger A and Giladi N: The LRRK2 G2019S mutation as the cause of Parkinson's disease in Ashkenazi Jews. J Neural Transm 116: 1473-1482, 2009.

28. Kumar S, Bhatia M and Behari M: Sleep disorders in Parkinson's disease. Mov Disord 17: 775-781, 2002.

29. Stacy M: Sleep disorders in Parkinson's disease: epidemiology and management. Drugs Aging 19: 733-739, 2002.

30. Friedman A: Sleep pattern in Parkinson's disease. Acta Med Pol 21: 193-199, 1980

31. Friedman JH and Chou KL: Sleep and fatigue in Parkinson's disease. Parkinsonism Relat Disord 10 (Suppl 1): S27-S35, 2004.

32. Yu SY, Sun L, Liu Z, Huang XY, Zuo LJ, Cao CJ, Zhang W and Wang XM: Sleep disorders in Parkinson's disease: Clinical features, iron metabolism and related mechanism. PLoS One 8 e82924, 2013.
33. Cirelli C: The genetic and molecular regulation of sleep: From fruit flies to humans. Nat Rev Neurosci 10: 549-560, 2009.

34. Comella CL, Tanner CM and Ristanovic RK: Polysomnographic sleep measures in Parkinson's disease patients with treatment-induced hallucinations. Ann Neurol 34: 710-714, 1993

35. Ozekmekçi S, Apaydin H and Kiliç E: Clinical features of 35 patients with Parkinson's disease displaying REM behavior disorder. Clin Neurol Neurosurg 107: 306-309, 2005.

36. Poryazova R, Oberholzer M, Baumann CR and Bassetti CL: REM sleep behavior disorder in Parkinson's disease: A questionnaire-based survey. J Clin Sleep Med 9: 55-59A, 2013

37. Piccoli G, Condliffe SB, Bauer M, Giesert F, Boldt K, De Astis S, Meixner A, Sarioglu H, Vogt-Weisenhorn DM, Wurst W, et al: LRRK2 controls synaptic vesicle storage and mobilization within the recycling pool. J Neurosci 31: 2225-2237, 2011.

38. Tononi G and Cirelli C: Sleep and synaptic homeostasis: A hypothesis. Brain Res Bull 62: 143-150, 2003.

39. Rosales-Corral SA, Acuña-Castroviejo D, Coto-Montes A, Boga JA, Manchester LC, Fuentes-Broto L, Korkmaz A, Ma S, Tan DX and Reiter RJ: Alzheimer's disease: Pathological mechanisms and the beneficial role of melatonin. J Pineal Res 52: 167-202, 2012.

40. Tarasenko A, Krupko O and Himmelreich N: Reactive oxygen species induced by presynaptic glutamate receptor activation is involved in $[(3) \mathrm{H}] \mathrm{GABA}$ release from rat brain cortical nerve terminals. Neurochem Int 61: 1044-1051, 2012.

41. Giniatullin AR, Darios F, Shakirzyanova A, Davletov B and Giniatullin R: SNAP25 is a pre-synaptic target for the depressant action of reactive oxygen species on transmitter release. J Neurochem 98: 1789-1797, 2006 